\title{
CONTRIBUIÇÕES DA TERAPIA COGNITIVO COMPORTAMENTAL NO TRATAMENTO DA OBESIDADE
}

\author{
Ana Raquel Mattoso Chagas Martins \\ Graduanda em Psicologia/ISECENSA/RJ \\ anaraquelmattoso@yahoo.com.br \\ Alice Pessanha Rocha de Assis \\ Graduanda em Psicologia/ISECENSA/RJ \\ alice-assis@uol.com.br \\ Amanda Barbosa de Freitas \\ Graduanda em Psicologia/ISECENSA/RJ \\ dynhahdefreitas@gmail.com
}

\author{
Bianca Machado da Silva Rangel \\ Graduanda em Psicologia/ISECENSA/RJ \\ bibimsr@ hotmail.com
}

\section{Larissa Barreto Bastos}

Graduando em Psicologia/ISECENSA/RJ

Psicologia2014lala@gmail.com

\section{Erika Costa Barreto Monteiro de Barros \\ Mestre em Cognição e Linguagem/UENF/RJ \\ erikacostabarreto@yahoo.com.br}

\section{RESUMO}

Levando em consideração que o homem é o único animal capaz de modificar seu corpo através do pensamento, a Terapia Cognitivo Comportamental (TCC) vem se configurando como um importante aliado no combate à obesidade, proporcionando uma reestruturação cognitiva capaz de modificar não apenas um corpo obeso, mas todo um estilo de vida. A (TCC) tem sido amplamente usada no tratamento da obesidade em associação com outras intervenções, como o acompanhamento nutricional e a prática de atividade física. Apresenta duração breve e com sessões estruturadas nas quais o paciente é solicitado a colaborar ativamente na identificação e na mudança de seus comportamentos e sentimentos que contribuem para a obesidade. $\mathrm{O}$ objetivo do presente trabalho consiste em verificar a eficácia do apoio psicoterápico com base na Terapia Cognitivo Comportamental na redução de peso de pacientes obesos.

Palavras-chave: Corpo; Peso; Tratamento. 


\title{
CENTRO EDUCACIONAL NOSSA SENHORA AUXILIADORA \\ INSTITUTOS SUPERIORES DE ENSINO DO CENSA \\ CENTRO DE PESQUISA E PÓS-GRADUAÇÃO
}

\begin{abstract}
Considering that man is the only animal able to modify your body through thought, Cognitive Behavioral Therapy (CBT) is shaping up as an important ally in the fight against obesity, providing a cognitive restructuring capable of modifying not only an obese body but change a whole lifestyle. Cognitive behavioral therapy (CBT) has been widely used in the treatment of obesity in combination with other interventions, such as nutritional counseling and physical activity. CBT is a short term therapy and structured sessions in which the patient is asked to actively collaborate in identifying and changing their behaviors and feelings that contribute to obesity. The aim of this study is to verify the effectiveness of psychotherapeutic support based on Cognitive Behavioral Therapy in reducing weight of obese patients.
\end{abstract}

Keywords: Body; Weight; Treatment.

\section{INTRODUÇÃO}

Nas últimas décadas é notório e significativo o aumento na prevalência de indivíduos obesos na sociedade, sua magnitude e velocidade de evolução em vários países do mundo, classifica a obesidade como uma pandemia, tão elevada que a OMS considera esta doença como epidemia global do século XXI estando presente em países desenvolvidos como também em países em desenvolvimento, dentre eles no Brasil.

O panorama mundial e brasileiro de doenças crônicas não transmissíveis tem revelado um desafio para a saúde pública, onde crescem a prevalência de obesos e sobrepesos nos últimos 30 anos.

Falar em obesidade é também falar sobre a saúde física e mental, um fator importante no desenvolvimento individual. Entretanto, observa-se que a obesidade é tratada pela maior parte das pessoas como um fator estético esquecendo que o ponto crucial é a vida, e dela dependem a saúde física e mental. Esta epidemia causa um forte impacto na saúde e bem-estar das populações assim como na economia das nações. Preocupados, a Organização Mundial da Saúde (OMS), órgão de máxima autoridade em saúde, relatou em 2012 que meio bilhão de pessoas, 12\% da população está obesa. (NEUFELD, MOREIRA, XAVIER, 2012)

Se em algum momento da história o obeso foi tido como próspero e saudável, deixou de ser assim considerado no mundo contemporâneo. A correlação entre obesidade, complicações orgânicas e mortalidade conferiu ao obeso o status de doente e a obesidade o de doença que precisa ser tratada.

A modernização mundial trouxe mudanças econômicas, sociais e demográficas, responsáveis pela maior oferta de alimentos, como a transição nutricional, caracterizada pelo aumento do consumo de açucares, gorduras, cereais refinados e pela redução no consumo de carboidratos complexos e fontes de fibras. Os fatores socioculturais também influenciam como causas para a obesidade, o nível socioeconômico interfere no acesso a informação e na disponibilidade de alimentos, determinando mais uma vez a prevalência para a obesidade.

Perder peso não é um trabalho fácil, requer mudança de hábito, bem como manter essa perda de peso. Por isto, trabalhar os aspectos emocionais são fundamentais com indivíduos com obesidade. 


\section{CENTRO EDUCACIONAL NOSSA SENHORA AUXILIADORA \\ INSTITUTOS SUPERIORES DE ENSINO DO CENSA \\ CENTRO DE PESQUISA E PÓS-GRADUAÇÃO}

O presente trabalho busca investigar se tratamento com intervenção em TCC proporciona o emagrecimento eficaz e a manutenção em longo prazo da melhora alcançada e não somente temporária. É de nosso interesse investigar se os pacientes submetidos a sessões de terapia grupal com enforque na Terapia Cognitivo Comportamental apresentam resultados relevantes em relação ao emagrecimento do que aqueles que não têm esse apoio na luta contra a balança.

\section{METODOLOGIA}

Até o presente momento fizemos um levantamento teórico capaz de embasar a prática em sessões de terapia grupal com enfoque na Terapia Cognitivo Comportamental. Redigimos o Termo de Consentimento Livre Esclarecido e obtivemos recentemente a aprovação do Comitê de Ética para iniciarmos nossa intervenção. Elaborarmos ainda um questionário de triagem para a seleção de candidatos para participarem da pesquisa, que já estão sendo distribuídos entre possíveis participantes. Nosso próximo passo será a triagem dos candidatos, a separação dos mesmos entre grupo controle e grupo de intervenção para que possamos então, começar as análises que nos levarão à conclusão desta pesquisa.

\section{CONSIDERAÇÕES FINAIS}

A literatura aponta para a necessidade que o mundo contemporâneo apresenta de pensarmos a obesidade, não apenas como uma doença médica a ser tratada apenas com intervenções médicas, mas como uma realidade epidemiológica que retrata aspectos sociais e culturais típicos de uma era.

Sendo assim, pensar o tratamento da obesidade como uma patologia que carece da interdisciplinaridade, visto que suas causas são múltiplas e seu tratamento assim também deve ser, nos leva a refletir sobre a subjetividade das pessoas que precisam perder peso, bem como a forma de diagnosticar, tratar e lidar com esse indivíduo.

Além dos aspectos bioéticos envolvidos, como a formação de vínculo entre profissional e paciente para que a adesão ao tratamento ocorra com sucesso, a forma de lidar com o diagnóstico e apresentação do plano de tratamento, analisar os fatores culturais e comunitários são de extrema relevância, bem como promover a liberdade do paciente frente a escolha de mudar suas atitudes e modo de ser sem que isso reflita num sentimento de abandono.

Nesse sentido, a orientação familiar e da rede de pertinência, aliado a técnicas cógnito comportamentais que partem da premissa da identificação de pensamentos disfuncionais e remissão das crenças centrais e intermediárias, evidenciam-se como importantes ferramentas no tratamento psicológico da obesidade, que através da metodologia focada na cooperação entre o terapeuta e o cliente, age de forma que as estratégias para superação dos problemas seja planejada em conjunto.

O monitoramento de hábitos alimentares, o treino de resoluções de problemas, são técnicas da TCC que fazem a diferença no processo psicoterápico fazendo com que a pessoa aprenda como agir quando estiver estressado, cansado, doente, chateado, aborrecido ou sem ter o que fazer. E conseguirá controlar não só a sua alimentação e o seu peso, mas controlará melhor a sua vida também. 


\section{CENTRO EDUCACIONAL NOSSA SENHORA AUXILIADORA \\ INSTITUTOS SUPERIORES DE ENSINO DO CENSA \\ CENTRO DE PESQUISA E PÓS-GRADUAÇÃO}

\section{REFERÊNCIAS}

BAHLS, S. C.; NAVOLAR, A.; BASSETTI B. Terapia Cognitivo - Comportamentais: Conceitos e Pressupostos Teóricos, 2004. Pesquisado: Acessado em: 23.05.2015

BRITO, C.L.S, BYSTRONSKI D.P., MOACH K.D., STENZEL L.M., REPETTO G. Obesidade: Terapia Cognitivo-Comportamental. Sociedade Brasileira de Endocrinologia e Metabologia/Projetos Diretrizes. 2005. Pesquisado: http://www.projetodiretrizes.org.br/5_volume/34-obesterap.pdf. Acessado: 29.11.2014

DUCHESNE, MONICA. O consenso latino-americano em obesidade. Rev. bras. ter. comport. cogn. vol.3 no.2 São Paulo dez. 2001

GONZALEZ, A.; LIRA, G.; CASTRO. T. Formas de tratamento do sobrepeso ou obesidade utilizadas por estudantes de ensino médio de uma escola pública de Salvador, Bahia. Revista Brasileira de Obesidade, Nutrição e Emagrecimento, São Paulo v.2, n. 11, p.446-466, Set/Out. 2008. ISSN 1981-9919.

NEUFELD, C. B.; MOREIRA, C. A. P. M.; XAVIER, G.S. Terapia Cognitiva- Comportamental em Grupos de Emagrecimento: O relato de Uma Experiência. Porto Alegre-RS. Revista PSICO, v. 43, n. 1, pp. 93-100, jan./mar. 2012.

RANGÉ, B. P.; FALCONE, E. M. DE O.; SARDINHA, A. História e panorama atual das terapias cognitivas no Brasil. REVISTA BRASILEIRA DE TERAPIAS COGNITIVAS, 2007. Pesquisado:< http://rbtc.org.br/edicao_atual.asp.> Acessado em: 09.04.2015.

RONDINA, R. DE C. Terapia Cognitivo Comportamental: Desenvolvimento Histórico, Tendências Atuais, 2006. Pesquisado: <http://www.revista.inf.br/psicologia11/pages/artigos/edic11anoVInov2008artigo05.pdf.> Acessado em 23.04.13.

VASQUES, F.; MARTINS, F. C.; DE AZEVEDO, A. P. Aspectos psiquiátricos do tratamento da obesidade, 2004. $\quad$ Pesquisado: $<\quad \mathrm{http}: / /$ www.scielo.br/scielo.php?pid=S0101 60832004000400013\&script=sci_arttext.> Acessado em: 29.11.2014.

SERRA, A. M.; MARTINS. Terapia Cognitiva, Terapia Cognitivo-Comportamental e Terapia Comportamental, 2013 Pesquisado:< http://www.itcbr.com/artigo_drana_tc.shtml.> Acessado em: 23.05.15.

SUASTIKA, K. Update in the managemente of obesity. Acta Med Indones. V.38, n.4, Oct-Dec, p. 231-7. 2006.

WORLD HEALTH ORGANIZATION Obesity: preventing and managing the global epidemic reporto f a WHO consultation on Obesity. Geneva 1998. 